\title{
A strategy to simplify the dynamic model of 6-DOF motion simulator
}

\author{
Liyi Yin ${ }^{1,}$, Chifu Yang ${ }^{1}$, Changhong Gao ${ }^{1}$, Dacheng Cong ${ }^{1}$, and Junwei Han ${ }^{1}$ \\ ${ }^{1}$ Harbin Institute of Technology, 92 Xidazhi Street, Harbin, Heilongjiang, 150001, P. R. China \\ ayinliyi@hit.edu.cn
}

Keywords: Motion simulator; Dynamic model; Movement decomposition; Model simplification Abstract. Highly nonlinearity and strong dynamic coupling exist in 6-DOF motion simulator. Usually, in order to make the system has good dynamic and static quality, a good controller need to be designed according to the nature of the dynamic model. Therefore, the research about the simplified dynamic model used for real-time solution will be very meaningful. First, KANE method is used to build complete compact multi-rigid-body dynamic model. Second, rigid body and movement decomposition method are used to split dynamic model. On this basis, simplified strategy of the dynamic model of 6-DOF motion simulator based on dynamic contribution factor is proposed. Finally, the simulation verifies that the simplified dynamic model can be real-time calculated with high efficiency.

\section{Introduction}

6 DOF parallel mechanism has been widely used in the field of motion environmental simulation, because of its high accuracy, large load and fast moving capability [1-3]. However, hydraulic driven 6 DOF motion simulators have a relatively higher nonlinearity and stronger coupling property $[4,5]$. In order to provide the system with better dynamic and static quality, a good designed controller based on dynamic model is needed. Generally the dynamic modelling and analysis methods include Newton Euler method, Lagrangian method, principle of virtual work, Kane method, and so on [6-9]. Although they are different from each other, under the same given condition, the models of rigid body dynamics are equivalent. The difference appears on the complexity of the modelling procedure and the computational efficiency of the final model. For achieving a simplified dynamic model of real-time solving, investigations of dynamic modelling have been carried out by many scholars on parallel mechanisms. Chaoui et al analysed the positive definiteness of mass matrix and its symmetry, as well as the mathematical character of Coriolis force / centrifugal force term and gravity term [10]. Jiang et al has come with a structural optimal design method based on the inverse mass matrix property on the hinge joint space of a 6 DOF parallel mechanism[11]. Based on Lagrangian method, Abdellatif et al investigated the computing efficiency of inverse dynamic of a Stewart platform[12].

\section{Rigid body dynamic modelling of 6-DOF motion simulator based on KANE method}

6-DOF motion simulators include a motion platform, a fixed platform and six electro hydraulic servo systems. As shown in Fig. 1, pose tracking of the motion platform is realized through driving the concertina movement of the six hydraulic cylinders. For analyzing the kinetic and dynamic properties of the motion simulator, the barycenter of the motion platform which includes the load and upper hinge joints is defined as the origin of the body coordinate system. In the initial attitude, the inertial frame coincides with the body frame. The coordinate system is defined as shown in Fig. 2. OL is the origin of the inertial frame and OL-XL-YL-ZL is the inertial frame $\{\mathrm{L}\}$, which is relatively constant with the fixed platform; $\mathrm{OB}$ is the origin of the body frame, and $\mathrm{OB}-\mathrm{XB}-\mathrm{YB}-\mathrm{ZB}$ is the body frame $\{\mathrm{B}\}$, which is fixed on the motion platform. ai is the upper hinge point, bi is the lower hinge point, lli is the length of hydraulic leg. 


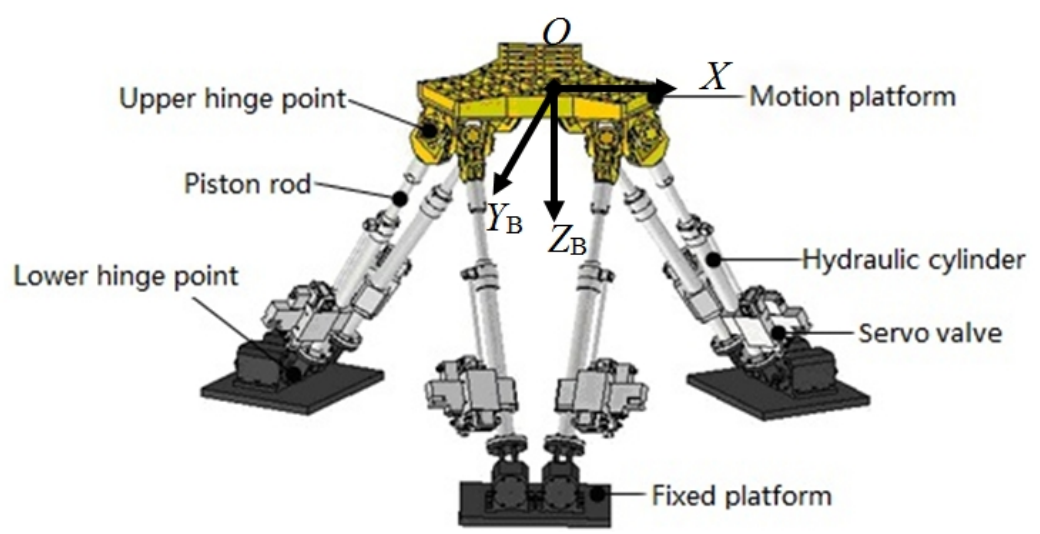

Fig.1 Configuration of motion simulator
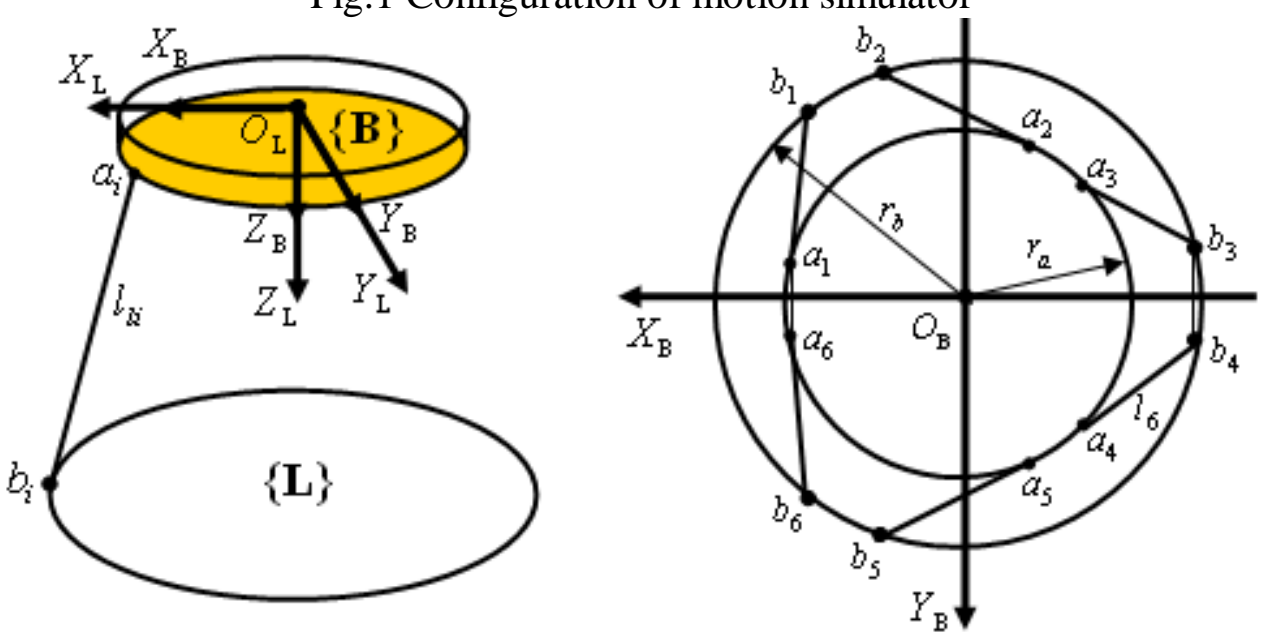

Fig.2 Definition of Cartesian coordinate system of docking motion simulator

The motion simulator can be treated as 13 rigid body systems, which include 6 hydraulic pistons and rods, 6 hydraulic cylinders and a platform with its load. According to the Newton's law of motion and the angular momentum theorem, the inertia force/torque of the motion simulator can be described as:

$$
\begin{aligned}
& \boldsymbol{F}_{p}=m_{p} \& \\
& \boldsymbol{M}_{p}=\boldsymbol{I}_{p} \boldsymbol{\omega}+\boldsymbol{\omega}^{L} \times \boldsymbol{I}_{p} \boldsymbol{\omega}^{L}
\end{aligned}
$$

Where, $m_{p}$ is the mass of platform and its load (unit: $\mathrm{Kg}$ ), $\boldsymbol{I}_{p}$ is the inertia tensor described in the inertial frame, and $\boldsymbol{I}_{p}=\boldsymbol{R} \boldsymbol{I}_{p}^{p} \boldsymbol{R}^{T}$.

In similar way, the inertia force/torque of the landing leg of this motion simulator can be described as:

$$
\begin{aligned}
& \boldsymbol{F}_{l v i}=\boldsymbol{J}_{a i, \bar{q}}{ }^{T}\left(\boldsymbol{J}_{a c i, a i}{ }^{T} m_{a}{ }_{a c i} \boldsymbol{\alpha}_{a c i}+\boldsymbol{J}_{b c i, a i}{ }^{T} m_{b} \boldsymbol{\alpha}_{b c i}\right) \\
& \boldsymbol{F}_{l a i}=\boldsymbol{J}_{a i, \bar{q}}{ }^{T} \boldsymbol{J}_{w i, a i}{ }^{T}\left(\left(\boldsymbol{I}_{a i}+\boldsymbol{I}_{b i}\right) \boldsymbol{\alpha}_{i i}+\boldsymbol{\omega}_{l i} \times\left(\boldsymbol{I}_{a i}+\boldsymbol{I}_{b i}\right) \boldsymbol{\omega}_{l i}\right)
\end{aligned}
$$

Where, $m_{a}$ and $m_{b}$ are the mass of piston and hydraulic cylinder respectively (unit: $\mathrm{Kg}$ ), $\boldsymbol{I}_{a i}, \boldsymbol{I}_{b i}$ are the inertia tensors described in the inertial frame of piston and hydraulic cylinder respectively, $\boldsymbol{J}_{w i, a i}$ is the Jacobian matrix between speed of upper hinge joint and the angular velocity of the leg, $\omega_{l i}$ is the rotational angular velocity of the leg, $\boldsymbol{J}_{a i, \bar{q}}$ is the Jacobian matrix between generalized velocity of the motion simulator and the speed of the upper hinge joint, $\boldsymbol{J}_{a c i, a i}, \boldsymbol{J}_{b c i, a i}$ are the Jacobian matrixes between the speed of upper hinge joint and the velocity of barycenter of piston and hydraulic cylinder respectively.

$$
\boldsymbol{\omega}_{l i}=\boldsymbol{J}_{w i, a i} \boldsymbol{v}_{a i}=\boldsymbol{J}_{w i, a i} \boldsymbol{J}_{a i, \bar{q}} \boldsymbol{q}^{\alpha}
$$


The generalized positive acting force of the motion simulator is $\tau$, which can be described as:

$$
\boldsymbol{\tau}=\boldsymbol{J}_{\overline{l q}}{ }^{T} \boldsymbol{F}_{a}
$$

Where, $\boldsymbol{F}_{a}$ is the net output force (unit: N).

Based on the Kane method, the multi-rigid body dynamic model of the motion simulator can be described as:

$$
\boldsymbol{M}(\boldsymbol{q}) \boldsymbol{C}(\boldsymbol{q}, \boldsymbol{q})^{q} \boldsymbol{q} \boldsymbol{q}(\boldsymbol{q})=\boldsymbol{\tau}
$$

Where, $\boldsymbol{M}(\boldsymbol{q})$ is the mass matrix, and $\boldsymbol{C}(\boldsymbol{q}, \hat{\boldsymbol{q}})$ is the Coriolis force, and $\boldsymbol{G}(\boldsymbol{q})$ is the gravity term.

$$
\begin{aligned}
& \boldsymbol{M}(q)=\left[\begin{array}{cc}
m_{p} \boldsymbol{I}_{3 \times 3} & 0 \\
0 & \boldsymbol{I}_{p}
\end{array}\right]+\sum_{i=1}^{6}\left\{\boldsymbol{J}_{a i, \bar{q}}{ }^{T}\left[\begin{array}{l}
\boldsymbol{J}_{a c i, a i}{ }^{T} m_{a} \boldsymbol{J}_{a c i, a i}+ \\
\boldsymbol{J}_{b c i, a i}{ }^{T} m_{b} \boldsymbol{J}_{b c i, a i}+ \\
\boldsymbol{J}_{w i, a i}{ }^{T}\left(\boldsymbol{I}_{a i}+\boldsymbol{I}_{b i}\right) \boldsymbol{J}_{w i, a i}
\end{array}\right] \boldsymbol{J}_{a i, \bar{q}}\right\} \\
& \boldsymbol{C}(\boldsymbol{q}, \overline{\boldsymbol{q}})=\left[\begin{array}{cc}
0_{3 x 3} & 0 \\
0 & \tilde{\boldsymbol{\omega}} \boldsymbol{I}_{p}
\end{array}\right]+\sum_{i=1}^{6}\left\{\begin{array}{c}
\boldsymbol{J}_{a i, \bar{q}}{ }^{T}\left[\begin{array}{l}
\boldsymbol{J}_{a c i, a i}{ }^{T} m_{a} d\left(\boldsymbol{J}_{a c i, a i} \boldsymbol{J}_{a i, \bar{q}}\right) / d t+ \\
\boldsymbol{J}_{b c i, a i}{ }^{T} m_{b} d\left(\boldsymbol{J}_{b c i, a i} \boldsymbol{J}_{a i, \bar{q}}\right) / d t
\end{array}\right]+ \\
\boldsymbol{J}_{a i, \bar{q}}{ }^{T} \boldsymbol{J}_{w i, a i}{ }^{T}\left[\begin{array}{l}
\left(\boldsymbol{I}_{a i}+\boldsymbol{I}_{b i}\right) d\left(\boldsymbol{J}_{w i, a i} \boldsymbol{J}_{a i, \bar{q}}\right) / d t+ \\
\boldsymbol{J}_{w i, a i} \boldsymbol{J}_{a i, \bar{q}} \hat{\boldsymbol{q}} \boldsymbol{T}\left(\boldsymbol{I}_{a i}+\boldsymbol{I}_{b i}\right) \boldsymbol{J}_{w i, a i} \boldsymbol{J}_{a i, \bar{q}}
\end{array}\right]
\end{array}\right\}
\end{aligned}
$$

In order to validate this mathematical model of multi rigid bodies, the models of the motion simulator are created through both SimMechanics and Simulink. From Fig. 3, the model of SimMechanics is created based on the physical and geometrical properties of the motion simulator, but the model from Simulink is in accordance with the mathematical Eq. 7. Under the same driven force, the motion output of the motion simulator model is given in Fig.4.

Fig. 4 tells that the simulation results of models from both Simulink and SimMechanics are coincident.
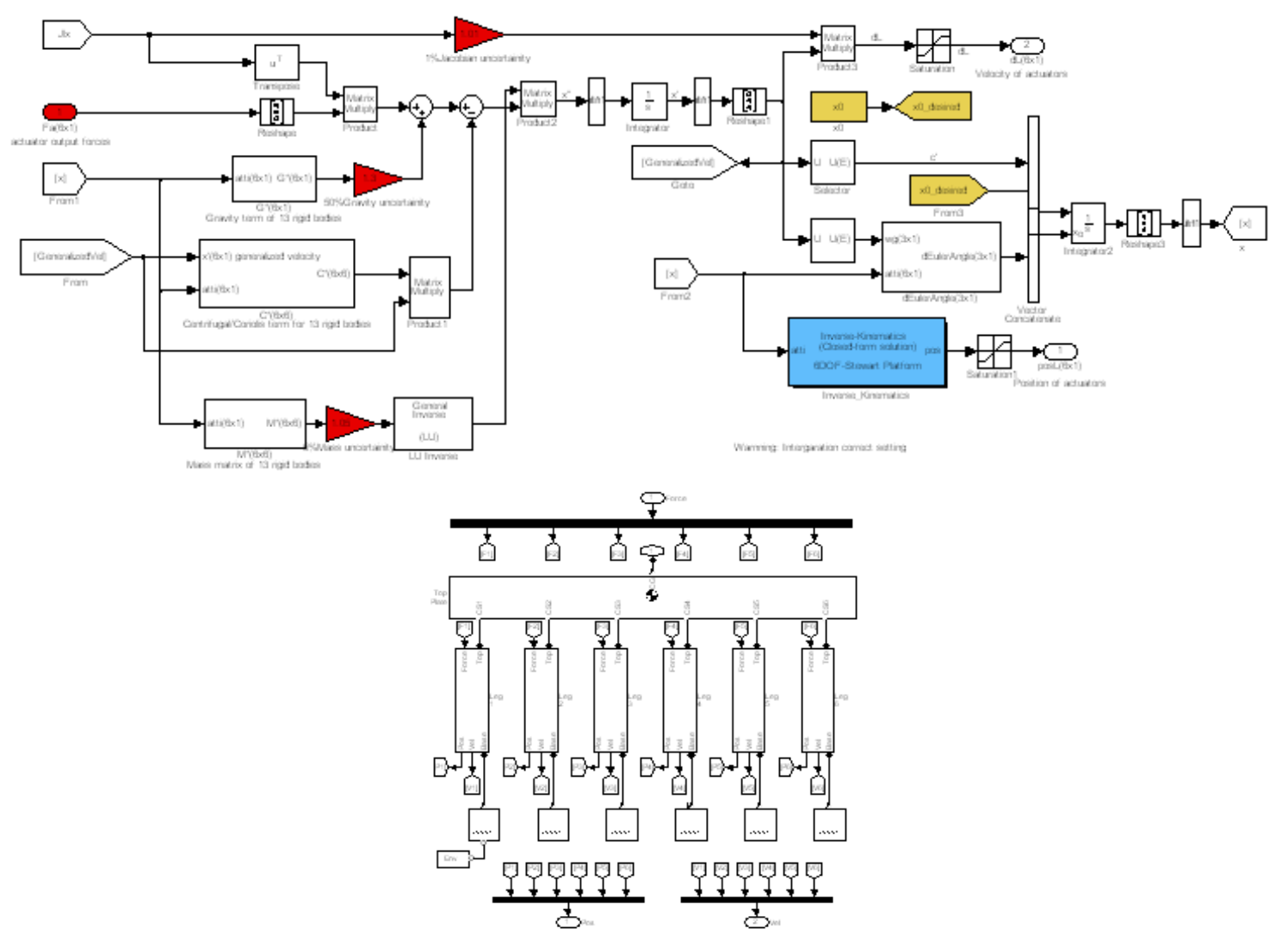

Fig3 Simulation models of parallel motion simulator 

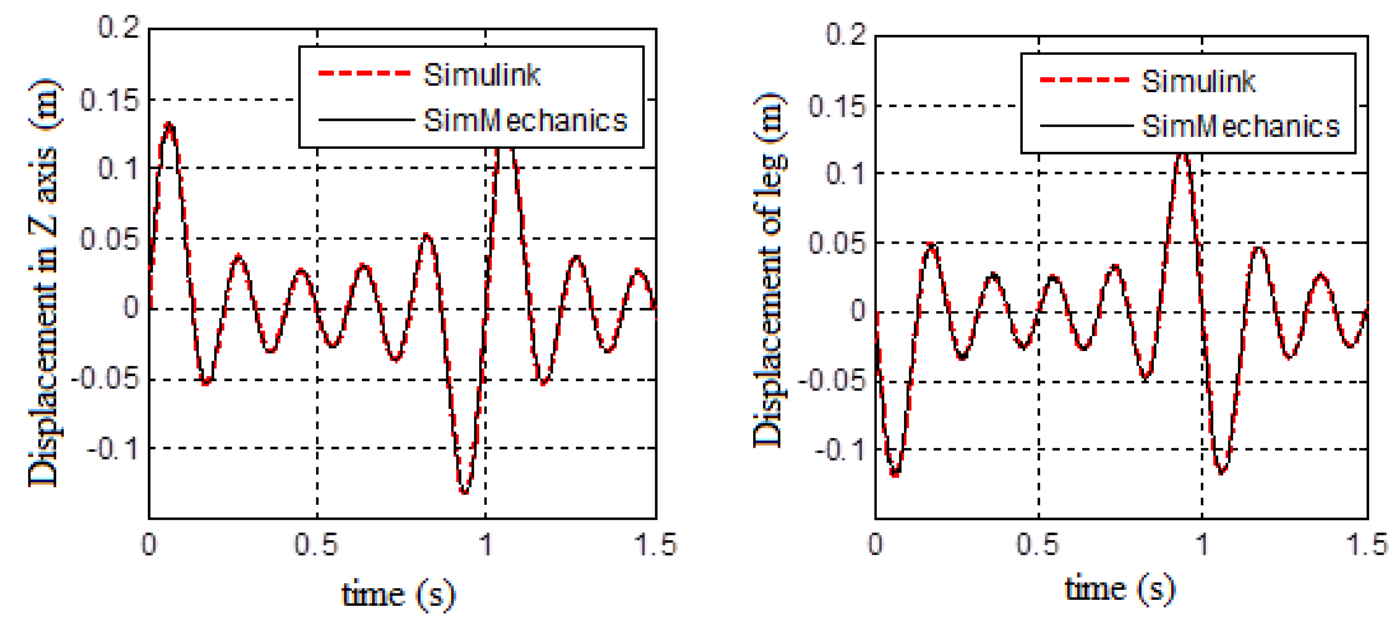

Fig.4 Simulation results of multi-rigid body dynamics of motion simulator

\section{Discretization of the rigid body dynamic model of 6 DOF motion simulators}

The motion simulator contains three main parts - rigid bodies of platform, piston rod and cylinder -. Therefore, the generalized inertia force of the motion mechanism can be resolved by rigid bodies as:

$$
\boldsymbol{F}^{*}=-\boldsymbol{\tau}=\boldsymbol{F}_{p}^{*}+\boldsymbol{F}_{r}^{*}+\boldsymbol{F}_{c}^{*}+\boldsymbol{G}^{*}
$$

Where, $\boldsymbol{F}_{r}^{*}$ is the generalized inertia force of piston $\operatorname{rod}, \boldsymbol{F}_{c}^{*}$ is the generalized inertia force of the cylinder, $\boldsymbol{F}_{p}^{*}$ is the generalized inertia force of platform, and $\boldsymbol{G}^{*}$ is the generalized gravity term.

$$
\begin{aligned}
\boldsymbol{F}_{p}^{*} & =-\boldsymbol{M}_{p} \boldsymbol{C}_{p} \boldsymbol{\Phi}^{\mathbf{\alpha}} \\
\boldsymbol{F}_{r}^{*} & =-\sum_{i=1}^{6}\left(\boldsymbol{J}_{a i, \bar{q}}{ }^{T} \boldsymbol{J}_{a c i a i}{ }^{T} \boldsymbol{F}_{l a i}+\boldsymbol{J}_{a i, \bar{q}}{ }^{T} \boldsymbol{J}_{w i, a i}{ }^{T} \boldsymbol{M}_{l a i}\right) \\
\boldsymbol{F}_{c}^{*} & =-\sum_{i=1}^{6}\left(\boldsymbol{J}_{a i, \bar{q}}{ }^{T} \boldsymbol{J}_{b c i, a i}{ }^{T} \boldsymbol{F}_{l b i}+\boldsymbol{J}_{a i, \bar{q}}{ }^{T} \boldsymbol{J}_{w i, a i}{ }^{T} \boldsymbol{M}_{l b i}\right) \\
\boldsymbol{G}^{*} & =-\boldsymbol{G}=-\left(\boldsymbol{G}_{p}+\boldsymbol{G}_{r}+\boldsymbol{G}_{c}\right)
\end{aligned}
$$

Where, $\boldsymbol{F}_{l a i}, \boldsymbol{F}_{l b i}$ are the generalized inertia forces of piston rod and cylinder respectively, and $\boldsymbol{M}_{l a i}, \boldsymbol{M}_{l b i}$ are the generalized inertia torques of piston rod and cylinder respectively, and $\boldsymbol{G}_{p}, \boldsymbol{G}_{r}, \boldsymbol{G}_{c}$ are the gravity terms of the platform, piston rod and cylinder respectively.

\section{The simplifying stratagem of the rigid body dynamic model of 6 DOF motion simulators}

The dynamic model simplification is developed by analysing the contribution of each dynamic term after the model decomposition. Therefore, the simplifying strategy could be different from each other and the simplification results can be affected by the structure of motion simulator, inertia parameters, the movement capability and the target for simplifying.

It is hard to achieve simplified model analysis method due to the dynamic model complexity, coupling characteristics between model parameters and variables. This article uses simulation analysis method of the dynamic contribution to achieve simplified models of motion simulation. In order to achieve computational efficiency and lower simplification errors which are suitable for high real-time control. As for dynamic model of simplified motion environmental mechanical structure, the aim of simplification is to set simplified accuracy below $8 \%$ and model execution time below $0.2 \mathrm{~ms}$.

The maximum capacity of movement of motion environmental mechanical structure can be classified by curves of maximum function into low, medium and high frequency band. The D1 is the maximum displacement and maximum speed points. D2 is the maximum speed and acceleration points. In order to analyze the contribution value of each dynamic item of motion environmental mechanical structure, the motion function based on the simplified analysis of the structures of D1 and D2 is shown below: 


$$
\begin{aligned}
& q_{1 c 1}=0.3 \sin (0.8333 t)[\mathrm{m}] \quad, \quad q_{2 c 1}=0.3 \sin (0.8333 t)[\mathrm{m}] \quad, \quad q_{3 c 1}=0.18 \sin (1.3889 t)[\mathrm{m}] \\
& q_{4 r 1}=19 \sin (1.053 t)[\mathrm{deg}] \quad, \quad q_{5 r 1}=18 \sin (1.111 t)[\mathrm{deg}] \quad, \quad q_{6 r 1}=30 \sin (0.667 t)[\mathrm{deg}] \quad \text {; } \\
& q_{1 c 2}=0.0064 \sin (39.2 t)[\mathrm{m}] \quad, \quad q_{2 c 2}=0.0064 \sin (39.2 t)[\mathrm{m}] \quad, \quad q_{3 c 2}=0.0064 \sin (39.2 t)[\mathrm{m}] \quad \text {, } \\
& q_{4 r 2}=10 \sin (2 t)[\mathrm{deg}] \quad, \quad q_{5 r 2}=10 \sin (2 t)[\mathrm{deg}] \quad, \quad q_{6 r 2}=10 \sin (2 t)[\mathrm{deg}] .
\end{aligned}
$$

The first step is to analyze the contribution of the dynamic items $\boldsymbol{F}_{a r}, \boldsymbol{F}_{a c}, \boldsymbol{F}_{a p}, \boldsymbol{F}_{a G p}, \boldsymbol{F}_{a G a}, \boldsymbol{F}_{a G b}$ by resolution of rigid in dynamic model of simplified motion environmental mechanical structure to holonomic dynamic model. $q_{1 \mathrm{c} 1}, q_{1 \mathrm{c} 2}, q_{2 \mathrm{c} 1} q_{2 \mathrm{c} 2}, q_{3 \mathrm{c} 1}, q_{3 \mathrm{c} 2}, q_{4 \mathrm{r} 1}, q_{4 \mathrm{r} 2}, q_{5 \mathrm{r} 1}, q_{5 \mathrm{r} 2}, q_{6 \mathrm{r} 1}, q_{6 \mathrm{r} 2}$ are input dynamic model of motion simulator separately, excitating the system. The maximum absolute value $\left|\boldsymbol{F}_{a r}\right|_{\max }$, $\left|\boldsymbol{F}_{a c}\right|_{\max },\left|\boldsymbol{F}_{a p}\right|_{\max },\left|\boldsymbol{F}_{a G p}\right|_{\max },\left|\boldsymbol{F}_{a G a}\right|_{\max }$, and $\left|\boldsymbol{F}_{a G b}\right|_{\max }$ of the dynamic item due to resolution of rigid are shown in Fig. 5 for an example. At D1, the maximum absolute values $\left|\boldsymbol{F}_{a r}\right|_{\max },\left|\boldsymbol{F}_{a c}\right|_{\max },\left|\boldsymbol{F}_{a p}\right|_{\max },\left|\boldsymbol{F}_{a G p}\right|_{\max }$, $\left|\boldsymbol{F}_{a G a}\right|_{\max }$, and $\left|\boldsymbol{F}_{a G b}\right|_{\max }$ of output force induced by translation are $9.2 \mathrm{~N}, 2.3 \mathrm{~N}, 26 \mathrm{~N}, 447 \mathrm{~N}, 242 \mathrm{~N}$, and $50 \mathrm{~N}$. At D2, the maximum absolute values of output force induced by translation are $395 \mathrm{~N}, 107 \mathrm{~N}$, $1148 \mathrm{~N}, 260 \mathrm{~N}, 155 \mathrm{~N}$, and $12.7 \mathrm{~N}$.

At both D1 and D2, the absolute values of output force induced by twirl are $9.5 \mathrm{~N}, 2.1 \mathrm{~N}, 23 \mathrm{~N}, 312 \mathrm{~N}$, $180 \mathrm{~N}$, and $23 \mathrm{~N}$. The percentage of contribution to holonomic dynamic of Platform, piston rods and cylinder is illustrated in Table 1.

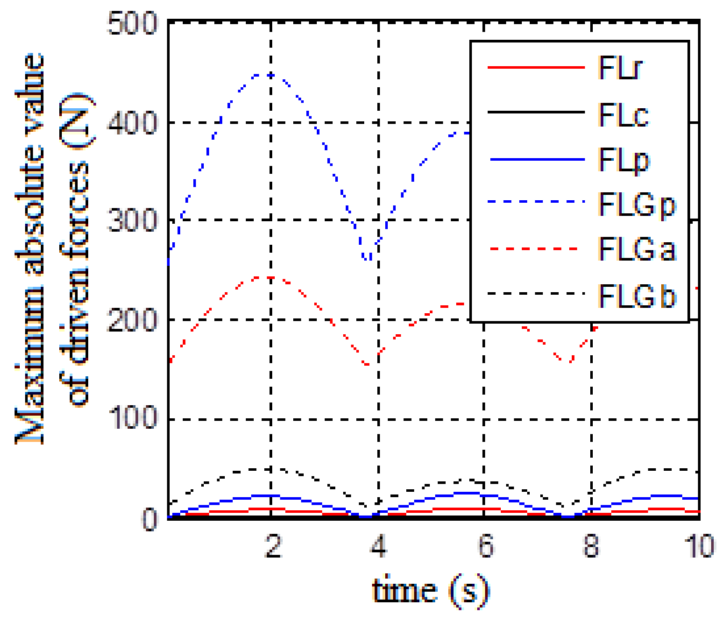

\begin{tabular}{|c|c|c|c|}
\hline $\begin{array}{l}\text { Dynamic } \\
\text { term }\end{array}$ & $\begin{array}{l}\text { Contribution ratio } \\
\text { from translational } \\
\text { motion at D1 }(\%)\end{array}$ & $\begin{array}{l}\text { Contribution ratio } \\
\text { from translational } \\
\text { motion at D2 }(\%)\end{array}$ & $\begin{array}{l}\text { Contribution ratio from } \\
\text { rotational motion at D1 } \\
\text { and D2 }(\%)\end{array}$ \\
\hline $\boldsymbol{F}_{a r}$ & 1.2 & 19.1 & 1.8 \\
\hline $\boldsymbol{F}_{a c}$ & 0.28 & 5.3 & 0.4 \\
\hline $\boldsymbol{F}_{a p}$ & 2.9 & 55.5 & 4.2 \\
\hline $\boldsymbol{F}_{a G p}$ & 58.6 & 30.7 & 59.6 \\
\hline $\boldsymbol{F}_{a G a}$ & 34.5 & 18.5 & 32.9 \\
\hline $\boldsymbol{F}_{a G b}$ & 6.6 & 1.4 & 6.7 \\
\hline
\end{tabular}

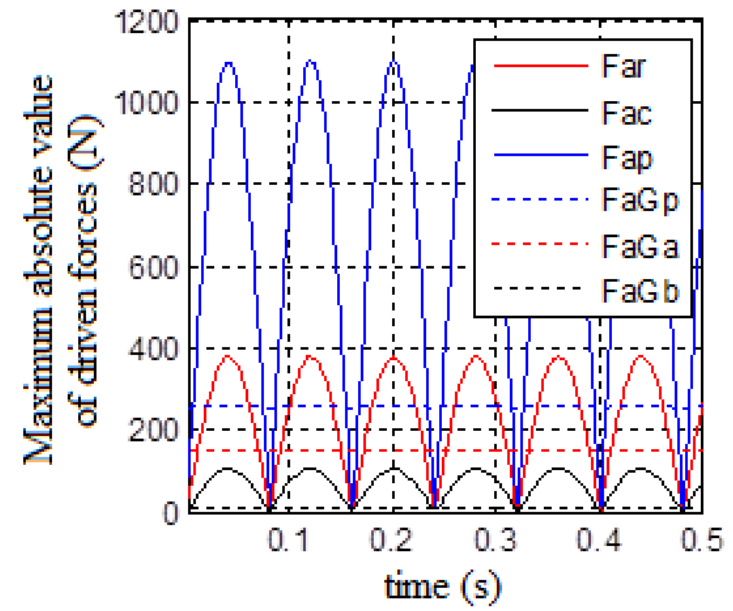

Fig.5 Driven forces of legs under $q_{1 \mathrm{c} 1}, q_{1 \mathrm{c} 2}$

Table 1 Contributions in percentage of each rigid body

It can be found in Fig. 5 and Table 1, that the contribution of platform to dynamic is maximum, followed by piston rod and then cylinder whether driving force is induced by gravity or motion environmental simulation structure. As driving force items $\boldsymbol{F}_{a r}, \boldsymbol{F}_{a c}, \boldsymbol{F}_{a p}$ induced by motion of system is ignorable during twirl and low frequency translation, holonomic dynamic model can be derived as followed:

$$
\boldsymbol{F}_{a}=\boldsymbol{F}_{a G p}+\boldsymbol{F}_{a G a}+\boldsymbol{F}_{a G b}
$$


The contribution of cylinder dynamic items $\boldsymbol{F}_{a c}, \boldsymbol{F}_{a G b}$ is ignorable during medium and high frequency translation, so the holonomic dynamic model can be derived as followed:

$$
\begin{aligned}
\boldsymbol{F}_{a} & =\boldsymbol{F}_{a r}+\boldsymbol{F}_{a p}+\boldsymbol{F}_{a G p}+\boldsymbol{F}_{a G a} \\
& =\left(\boldsymbol{F}_{a r v 11}+\boldsymbol{F}_{a r v I 2}+\boldsymbol{F}_{a r w l 1}+\boldsymbol{F}_{a r w I 2}+\boldsymbol{F}_{a r w I 3}\right)+\left(\boldsymbol{F}_{a p I 1}+\boldsymbol{F}_{a p I 2}\right)+\left(\boldsymbol{F}_{a G p}+\boldsymbol{F}_{a G a}\right)
\end{aligned}
$$

It can be also found that both simplified model (15) and (16) lessen the computational items in dynamic model of motion environmental simulation structure and shorten model execution time, but model (16) is still relatively complicated. In order to further simplify the model, simplified analysis method is adopted to analyze the contribution of rigid dynamic items $\boldsymbol{F}_{a p 11}, \boldsymbol{F}_{a p l 2}, \boldsymbol{F}_{a r v 11}, \boldsymbol{F}_{a r v l 2}, \boldsymbol{F}_{a r w l 1}, \boldsymbol{F}_{a r w I 2}$, and $\boldsymbol{F}_{\text {arwl3 }}$ based on resolution of motion. The contribution of dynamic items are illustrate as Table 2.

Table 2 Contributions in percentage of each dynamic term

\begin{tabular}{cccccccc}
\hline $\begin{array}{c}\text { Dynamic } \\
\text { term }\end{array}$ & $\boldsymbol{F}_{a p 11}$ & $\boldsymbol{F}_{\text {apl2 }}$ & $\boldsymbol{F}_{\text {arvl1 }}$ & $\boldsymbol{F}_{\text {arvl2 }}$ & $\boldsymbol{F}_{\text {arwl1 }}$ & $\boldsymbol{F}_{\text {arwl2 }}$ & $\boldsymbol{F}_{\text {arwl3 }}$ \\
\hline $\begin{array}{c}\text { Contribution } \\
\text { ratio (\%) }\end{array}$ & 55.5 & 0 & 18 & 0.2 & 1.1 & 0.02 & 0.03 \\
\hline
\end{tabular}

It can be found that the contribution of velocity-related dynamic items $\boldsymbol{F}_{a p l 2}, \boldsymbol{F}_{a r v I 2}, \boldsymbol{F}_{a r w I 2}, \boldsymbol{F}_{a r w 13}$ and related to angular acceleration of the piston $\operatorname{rod} \boldsymbol{F}_{a r w 11}$ is ignorable. Hence, equation (16) can be derived as:

$$
\boldsymbol{F}_{a}=\boldsymbol{F}_{a r v I 1}+\boldsymbol{F}_{a p I 1}+\boldsymbol{F}_{a G p}+\boldsymbol{F}_{a G a}
$$

Therefore, the dynamic model of motion environmental structure holonomic multi-rigid can be simplified by motion and frequency bands. Simplified model is (15) under the condition of rotation and low frequency translation; simplified model is (17) under the condition of medium and high frequency translation.

\section{The evaluation of a strategy to simplify of the dynamic model of 6-DOF motion simulator}

The accuracy and efficiency is the main index of model simplification, also the target of simplification. The dynamics model of simplified motion environmental mechanic structure is based on the previous method of model decomposition and simplification and the error of model which is describe via the ratio of the absolute value between the driving force of simplified and real model. Under the condition of the rotation and low frequency translation, the maximum error of the simplification is shown in the following Fig. 6 (a) and the error under condition of moderate and high frequency in Fig. 6 (b).

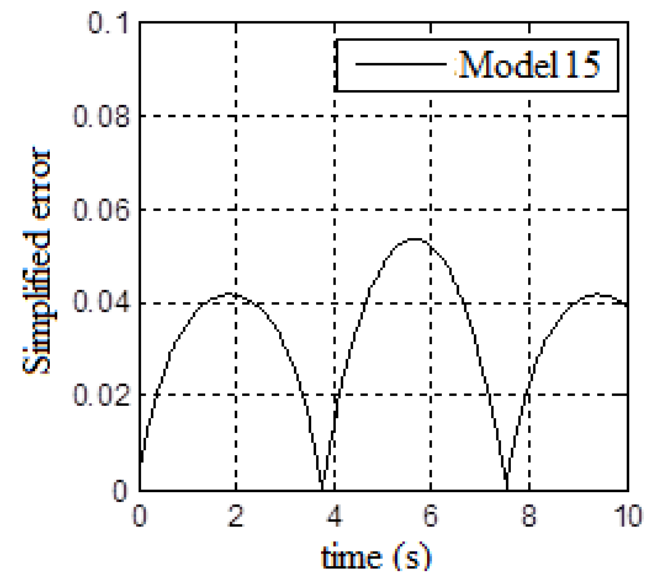

(a)

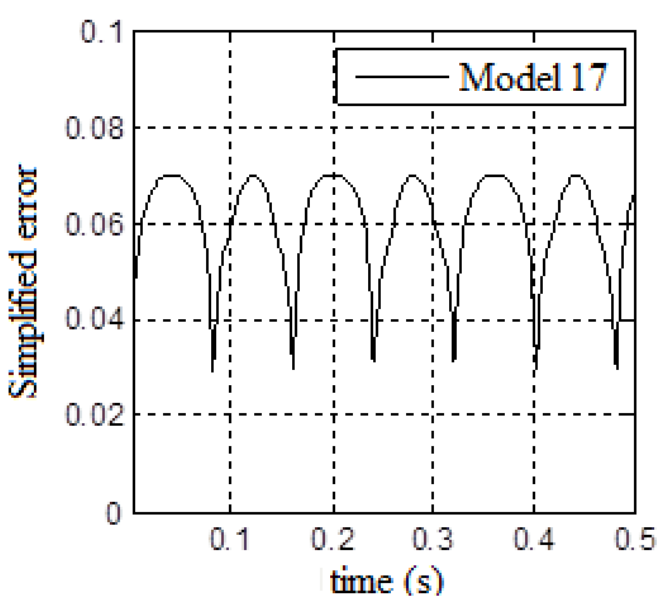

(b)

Fig.6 Simplified errors of dynamic model of parallel motion simulator

According to the chart, the error of rotation and low frequency translation is $5.8 \%$ while the error is $7.1 \%$ in the high frequency, the error of the aboving decomposition and simplification method is all below $8 \%$ as design. 
In terms of 6-DOF mechanical structure, it is common to ignore the dynamic effect of legs when design the structure or dynamic controller. Simplifying the parallel mechanism to the single rigid body dynamic model is widely applied in engineering and controller design. Especially, this method is fitted in particular motion or parallel mechanism which the mass of platform (including the load) is far above the mass of legs, in the other word, the legs contribute a little in dynamic.

$$
\boldsymbol{F}_{a}=\boldsymbol{F}_{a p}+\boldsymbol{F}_{a G p}
$$

Compared with single rigid body dynamic model, under different amplitudes and frequencies, the output of the motion simulator (15) and (17) is shown in 12(a)-(b) respectively.

According to Fig. 7, in the same situation, the dynamic model of motion environmental mechanic structure (17) the maximum simplification error is $32 \mathrm{~N}$. While the maximum error of single rigid body model (18) is $219 \mathrm{~N}$. The former method error is $12 \mathrm{~N}$ and the latter is $118 \mathrm{~N}$. Both errors of the former method are below $8 \%$, and the latter is above $30 \%$.

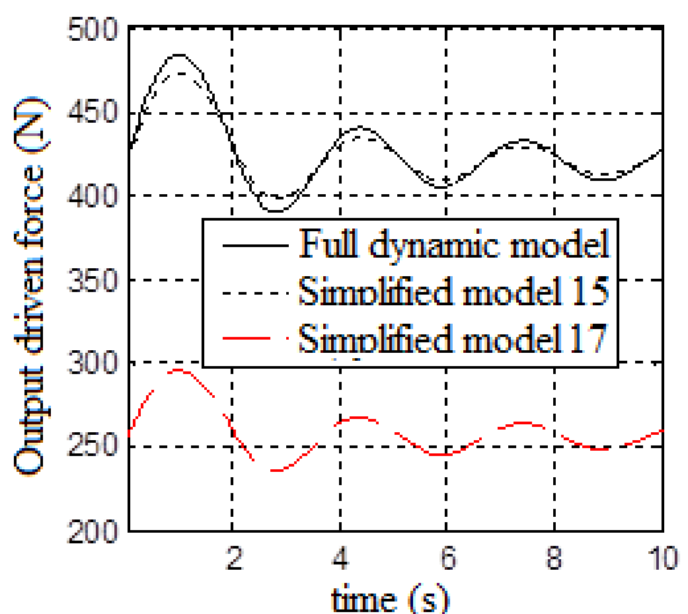

(a)

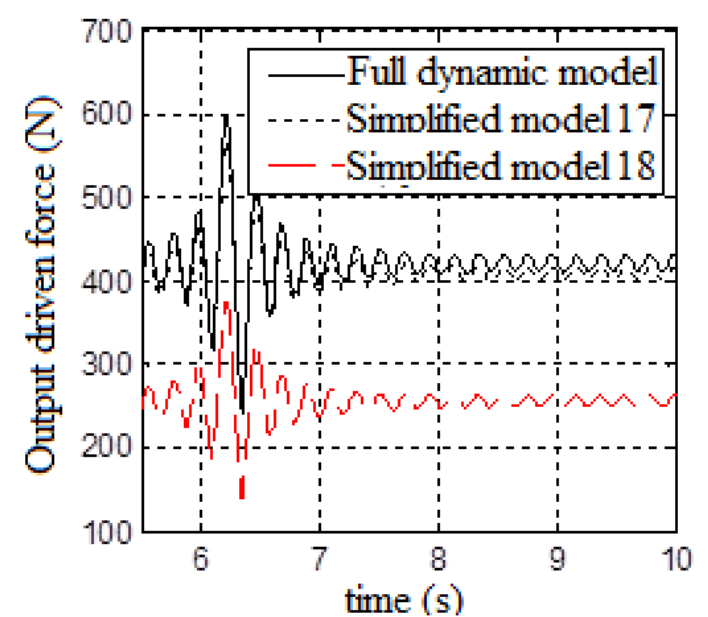

(b)

Fig.7 Output forces of dynamic model of parallel motion simulator

Experimental analysis to dynamic model of motion environmental simulation mechanical structure is conducted to evaluate the efficiency of simplified model. Homolomic model, simplified dynamic model (15) and (17) are encoded and executed on real-time computer to test computational time of dynamic model. Result is shown in Table 3.

Table 3 Executed time of dynamic model in real-time system

\begin{tabular}{lcll}
\hline Dynamic model & $\begin{array}{l}\text { Min/Max execution time } \\
(\mathrm{ms})\end{array}$ & $\begin{array}{l}\text { Average execution time } \\
(\mathrm{ms})\end{array}$ \\
\hline Simplified model (15) & $0.0090 / 0.0161$ & 0.0103 & \\
Simplified model (17) & $0.0553 / 0.0887$ & 0.0554 \\
Full dynamic model & $0.1874 / 0.3127$ & 0.2000 &
\end{tabular}

It can be found that the real-time computational efficiency of the dynamic model of motion environmental mechanical structure is increased by over $70 \%$ based on the condition that the simplified error is relatively low in simplified model derived by previous model resolution and simplification methodology. Therefore, dynamic model of motion environmental mechanical structure are suitable for the design of high real-time accuracy dynamic control system. In order to simplify environmental simulation structure dynamic characteristics analysis and design of controller, dynamic model of motion environmental simulation structure combining simplified model (15) and (17) can be derived below within the whole motion capability range:

$$
\boldsymbol{M}_{s l}(\boldsymbol{l}) \boldsymbol{G}_{l}(\boldsymbol{l})=\boldsymbol{F}_{a}
$$


In Eq. 19, $\boldsymbol{M}_{s l}(\cdot)$ refer to simplified metric of mass $\boldsymbol{M}_{s l}(\cdot)=\boldsymbol{J}_{l \bar{q}}{ }^{\mathrm{T}}\left(\boldsymbol{F}_{a r v I 1}+\boldsymbol{F}_{a p I 1}\right)$.

\section{Conclusion}

This article raises a simplified strategy of dynamic model toward the 6-DOF motion simulator structure. Firstly, the author builds multi-rigid dynamic model for integrity and simplicity of motion environmental simulation structure, and through SimMechanics and Simulink modeling simulation comparison to verify the correctness of the model respectively. Secondly, dynamic model is dispersed by the method of resolution of rigid and motion, based on which strategy to simplify mathematical model of dynamic contribution factor is proposed. High real-time computational efficiency of this strategy is proved by simulation.

\section{References}

[1] Stewart D. A platform with six degrees of freedom[J]. In Proc. Inst. Mech. Engrs., 1965, 180(15): 371-385.

[2] Merlet J P. Parallel manipulator: state of the art and perspectives[J]. Advanced Robotics, 1994, 8(6): 589-596.

[3] Yan H, Ye Z, Cong D. Space docking hybrid simulation prototype experiment system[J]. Chinese Journal of Mechanical Engineering, 2007, 43(9): 51.

[4] He J, Ye Z, Jiang H, et al. Coupling analysis based on joint-space model of parallel robot[J]. Chinese Journal of Mechanical Engineering, 2006, 42(6): 161-165.

[5] Wang W, Xie H, Fu X. Coupling characteristics of large hydraulic Stewart platform[J]. Chinese Journal of Mechanical Engineering, 2007, 43(9): 12.

[6] Dasgupta B, Mruthyunjaya T S. Closed-form dynamic equations of the general Stewart platform through the Newton-Euler approach[J]. Mechanism and Machine Theory, 1998, 33(7): 993-1012.

[7] Chen C T. A Lagrangian formulation in terms of Quasi-coordinates for the inverse dynamics of the general 6-6 Stewart platform manipulator[J]. JSME International Journal Series C: Mechanical Systems Machine Elements and Manufacturing, 2003, 46(3): 1084-1090.

[8] Tsai L W. Solving the inverse dynamics of a Stewart-Gough manipulator by the principle of virtual work[J]. ASME Journal of Mechanical Design, 2000, 122(1): 3-9.

[9] Liu M J, Li C X, Li C N. Dynamics analysis of the Gough-Stewart platform manipulator[J]. IEEE Transactions on Robotics and Automation, 2000, 16(1): 94-98.

[10] Chaoui H, Sicard P, Gueaieb W. ANN-Based adaptive control of robotic manipulator with friction and joint elasticity[J]. IEEE Transactions on Industrial Electronics, 2009, 56(8): 3174-3187.

[11] Jiang H Z, He J F, Tong ZZ. Characteristics analysis of joint space inverse mass matrix for the optimal design of a 6-DOF parallel manipulator[J]. Mechanism and Machine Theory, 2010, (45): 722-739.

[12] Abdellatif H, Heimann B. Computational efficient inverse dynamics of 6-DOF fully parallel manipulators by using the Lagrangian formalism[J]. Mechanism and Machine Theory, 2009, 44: 192-207. 